\title{
CONSISTENT LIMITATION METHODS
}

\author{
CASPER GOFFMAN AND G. M. PETERSEN
}

It is known that if a bounded sequence does not converge there are regular matrix methods which limit it to various values. It is thus pertinent to ask whether there is a collection $\mathfrak{A}$ of regular matrix methods which are consistent and such that every bounded sequence is limited by at least one method in the collection. No examples of such collections seem to have been published.

We define such a collection of methods and discuss its relation to other possible collections. ${ }^{1}$ The problem of finding all such collections seems to be extremely difficult.

A collection $\mathfrak{A}$ of methods is consistent if for every $A \in \mathfrak{A}, B \in \mathfrak{A}$, and bounded $\left\{s_{n}\right\}$ which is limited by both $A$ and $B$, we have $A \lim _{n \rightarrow \infty} s_{n}=B \lim _{n \rightarrow \infty} s_{n}$. We consider the collection $\mathfrak{A}$ of all methods $A=\left(a_{m n}\right)$ such that:

1. $A$ is regular.

2. $A$ is positive; i.e., $a_{m n} \geqq 0$ for all $m, n$.

3. For every $m$, there is a $k(m)$ such that $a_{m k(m)}=1 / 2$, and for every $n$ there is an $m$ such that $n=k(m)$; i.e., the number $1 / 2$ appears at least once in each row and in each column of $A$.

Lemma 1. The collection $\mathfrak{A}$ is consistent.

Proof. Let $\left\{s_{n}\right\}$ be a bounded sequence, let $A \in \mathfrak{A}$, and suppose $A \lim _{n \rightarrow \infty} s_{n}$ exists. We show that $A \lim _{n \rightarrow \infty} s_{n}=(u+l) / 2$, where $u=\lim \sup _{n \rightarrow \infty} s_{n}$ and $l=\lim \inf _{n \rightarrow \infty} s_{n}$. For, let $\left\{n_{\mu}\right\}$ and $\left\{n_{\mu}^{\prime}\right\}$ be increasing sequences for which $\lim _{n \rightarrow \infty} s_{n_{\mu}}=l$ and $\lim _{n \rightarrow \infty} s_{n^{\prime} \mu}=u$. Let $\left\{t_{n}\right\}$ be the $A$ transform of $\left\{s_{n}\right\}$. Define $m_{\mu}$ and $m_{\mu}^{\prime}$ so that $k\left(m_{\mu}\right)=n_{\mu}$ and $k\left(m_{\mu}^{\prime}\right)=n_{\mu}^{\prime}$, for $\mu=1,2, \cdots$. Then

$$
\begin{aligned}
\lim _{\mu \rightarrow \infty} t_{m_{\mu}} & =\lim _{\mu \rightarrow \infty} \sum_{n \neq n_{\mu}} a_{m_{\mu n} s_{n}}+\frac{l}{2} \\
& \leqq \lim _{\mu \rightarrow \infty} \sum_{n \neq n_{\mu}} a_{m_{\mu} n} u+\frac{l}{2} \leqq \frac{u+l}{2} .
\end{aligned}
$$

Presented to the Society, April 23, 1955 under the title $A$ class of consistent summability methods; received by the editors January 31, 1955 and, in revised form, May 29, 1955.

1 The discussion of such relations was suggested by the referee to whom we express our thanks. 
Similarly, $\lim _{\mu \rightarrow \infty} t_{m^{\prime}} \geqq(u+l) / 2$. Hence, if $\left\{s_{n}\right\}$ is $A$ limitable, then $A \lim _{n \rightarrow \infty} s_{n}=(u+l) / 2$. This shows that $\mathfrak{A}$ is consistent.

LEMMA 2. For every bounded $\left\{s_{n}\right\}$ there is an $A \in \mathfrak{A}$ such that $\left\{s_{n}\right\}$ is limited by $A$.

Proof. For every $m$, there are $c_{1} \geqq 0, c_{2} \geqq 0, c_{1}+c_{2}=1 / 2$, such that $\lim _{m \rightarrow \infty}\left(s_{m} / 2+c_{1} u+c_{2} l\right)=(u+l) / 2$. We define $A$ as follows:

$$
\begin{aligned}
a_{m m} & =1 / 2 & & \text { for all } m, \\
a_{m n_{m}} & =c_{1} & & \text { where }\left|s_{n_{m}}-\mathrm{u}\right|<1 / m \text { and } n_{m}>m, \\
a_{m n^{\prime} m} & =c_{2} & & \text { where }\left|s_{n^{\prime}}-l\right|<1 / m \text { and } n_{m}^{\prime}>m, \\
a_{m n} & =0 & & \text { for } n \neq m, n_{m}, n_{m}^{\prime} .
\end{aligned}
$$

Then $A \in \mathfrak{A}$ and $\left\{s_{n}\right\}$ is $A$ limitable.

We now have:

THEOREM 1. The collection $\mathfrak{A}$ is consistent and every bounded $\left\{s_{n}\right\}$ is limited by at least one $A \in \mathfrak{A}$.

We shall designate this limit by $\mathfrak{A} \lim _{n \rightarrow \infty} s_{n}$.

THEOREM 2. For any regular matrix $C$, there is a consistent collection $\mathfrak{B}$ of regular matrix methods, all consistent with $C$, which limit all bounded sequences.

Proof. Let $\mathfrak{B}$ consist of all $B=A C, A \in \mathfrak{A}$.

The collection $\mathfrak{A}$ in Theorem 1 is such that $\mathfrak{A} \lim _{n \rightarrow \infty} s_{n}=(u+l) / 2$, where $l=\lim \inf _{n \rightarrow \infty} s_{n}$ and $u=\lim \sup _{n \rightarrow \infty} s_{n}$. It is natural to ask whether, for every consistent collection $\mathfrak{B}$, which limits all bounded sequences, there is a function $f(l, u)$ such that $\mathfrak{B} \lim _{n \rightarrow \infty} s_{n}=f(l, u)$. That this is false follows from Theorem 2.

Corollary 1. There is a consistent collection $\mathfrak{B}$ which limits all bounded sequences such that for every $f(l, u)$ there is a bounded $\left\{s_{n}\right\}$ with $\Re \lim _{n \rightarrow \infty} s_{n} \neq f(l, u)$.

Proof. Let the matrix $C$ of Theorem 2 be the Cesàro $(C, 1)$ method and let $\left\{s_{n}\right\}=\{1,0,1,0, \cdots\}$ and $\left\{t_{n}\right\}=\{1,0,0,1,0,0, \cdots\}$. Then $f(l, u)$ is the same for both $\left\{s_{n}\right\}$ and $\left\{t_{n}\right\}$, but $\mathfrak{B} \lim _{n \rightarrow \infty} s_{n}=1 / 2$ and $\mathfrak{B} \lim _{n \rightarrow \infty} t_{n}=1 / 3$.

In contrast to this, we have:

TheOREM 3. If $\mathfrak{A}$ is any collection of consistent methods which limits all bounded sequences, and if the $\mathfrak{A}$ limit is given by a function $f(l, u)$, then $f(l, u)=(u+l) / 2$. 
Proof. Suppose $\mathfrak{A}$ and $f(l, u)$ satisfy the conditions of the theorem. Let $\left\{s_{n}\right\}=\{1,0,1,0, \cdots\}$ and suppose $\left\{s_{n}\right\}$ is limited by $A \in \mathfrak{A}$ to the value $k$. Then, for every $u \geqq 0,\left\{u s_{n}\right\}=\{u, 0, u, 0, \cdots\}$ is limited by $A$ to the value $k u$. Suppose $u \geqq l$. Since the sequences $\{u-l, 0, u-l, 0, \cdots\}$ and $\{l, l, l, \cdots\}$ are limited by $A$ to the values $k \cdot(u-l)$ and $l$, respectively, the sequence $\{u, l, u, l, \cdots\}$ is limited by $A$ to the value $k \cdot(u-l)+l=k u+(1-k) l$. Hence $f(l, u)$ $=k u+(1-k) l$.

It remains for us to show that $k=1 / 2$. We write $A=\left(a_{m n}\right)$ and observe that the $A$ limit of $\left\{s_{n}\right\}=\{1,0,1,0, \ldots\}$ is given by $\lim _{m \rightarrow \infty} \sum_{n=1}^{\infty} a_{m, 2 n-1}=k$, so that the $A$ limit of $\left\{t_{n}\right\}=\{0,1,0,1, \cdots\}$ is given by $\lim _{m \rightarrow \infty}^{\infty} \sum_{n=1}^{\infty} a_{m, 2 n}=1-k$. But, $A \lim _{n \rightarrow \infty} t_{n}=k u+(1-k) l$ $=k$, so that $1-k=k$ and $k=1 / 2$.

We wish to remark in closing that Theorem 2 indicates the difficulty of determining all consistent collections which limit all bounded sequences. In particular, we may ask the following question: If $\mathfrak{A}$ is a consistent collection of regular matrix methods, is there a consistent collection $\mathfrak{B}$ such that $\mathfrak{B} \supset \mathfrak{A}$ and every bounded sequence is limited by at least one $B \in \mathfrak{B}$ ?

The UNIVERsity OF OKLahoma 